\title{
Politiques de développement régional dans les Hautes Plaines occidentales algériennes : un bilan mitigé
}

Mohamed Hadeid

\section{(2) OpenEdition}

Journals

\section{Édition électronique}

URL : http://journals.openedition.org/developpementdurable/8190

DOI : 10.4000/developpementdurable.8190

ISSN : 1772-9971

Éditeur

Association DD\&T

\section{Référence électronique}

Mohamed Hadeid, «Politiques de développement régional dans les Hautes Plaines occidentales algériennes : un bilan mitigé », Développement durable et territoires [En ligne], Varia (2004-2010), mis en ligne le 26 mai 2009, consulté le 01 mai 2019. URL : http://journals.openedition.org/ developpementdurable/8190 ; DOI : 10.4000/developpementdurable.8190

Ce document a été généré automatiquement le 1 mai 2019.

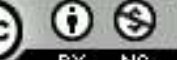

Développement Durable et Territoires est mis à disposition selon les termes de la licence Creative Commons Attribution - Pas d'Utilisation Commerciale 4.0 International. 


\title{
Politiques de développement régional dans les Hautes Plaines occidentales algériennes : un bilan mitigé
}

\author{
Mohamed Hadeid
}

1 L'Algérie a connu des mutations importantes grâce au programme de développement entrepris par l'Etat et ce, dès 1962, date à laquelle les Algériens ont mis fin à la colonisation française. L'indépendance est considérée comme une rupture à trois points de vue, politique d'abord, économique ensuite et spatiale enfin. L'Etat, en tant que pouvoir politique centralisé, s'est donné la primauté dans la conduite du projet de développement national en s'engageant dans une politique d'équilibre régional dans un but de redressement des inégalités et de dynamisation des économies affaiblies de certaines régions. Cette politique d'équilibre régional, bien plus que d'aménagement du territoire à proprement parler, explique certaines insuffisances enregistrées au niveau des politiques sectorielles de développement, notamment le délaissement relatif dont a souffert l'espace rural. Dans les Hautes Plaines steppiques occidentales, une des vastes régions rurales du pays, le mode de vie autrefois fondé sur le nomadisme et le pastoralisme a été fortement déstructuré par la colonisation tant socialement, qu'économiquement. Toutefois, malgré les efforts consentis depuis l'indépendance, elles demeurent toujours en marge du développement. Cela est-il lié à la société pastorale qui n'arrive pas à s'intégrer dans la politique étatique de développement ? Ou est-ce plutôt lié aux politiques de l'Etat qui n'ont pas su prendre en considération les spécificités de ce territoire?

D'une superficie d'environ $100000 \mathrm{~km}^{2}$, la région des Hautes Plaines occidentales occupe près de $4,2 \%$ du territoire national et plus de $45 \%$ des Hautes Plaines algériennes (la steppe algérienne). Cette vaste région abrite une population de faible densité ( $4 \mathrm{hab} / \mathrm{km}^{2}$ contre $15 \mathrm{hab} / \mathrm{km}^{2}$ pour le reste des Hautes Plaines algériennes) qui ne représente que $1.5 \%$ de la population algérienne. Cette population peu nombreuse se consacre 
essentiellement à l'élevage puisque la région abrite $12 \%$ du troupeau ovin algérien et plus du quart de celui de la steppe.

Figure 1. Limites naturelles des Hautes Plaines occidentales algériennes.

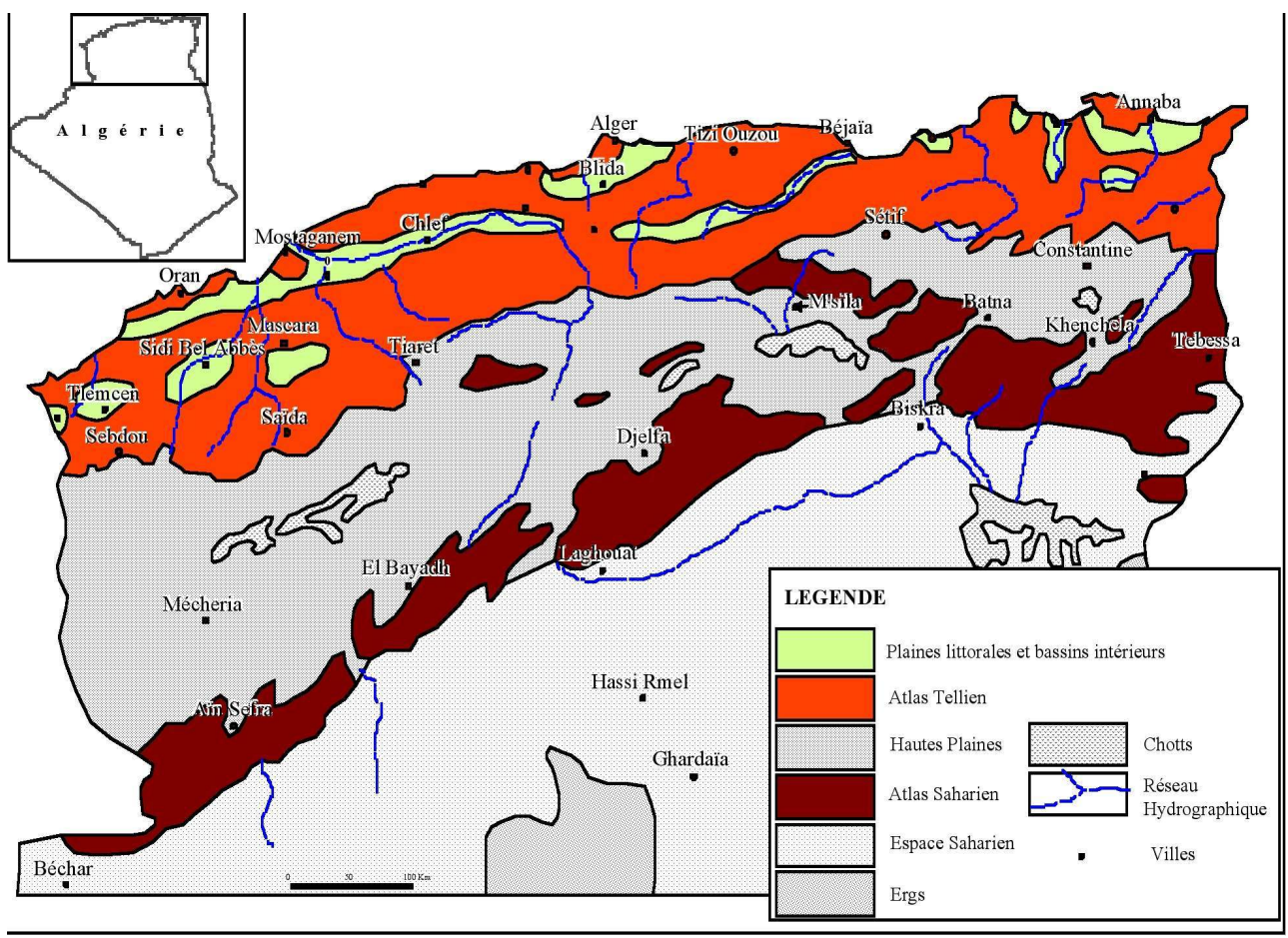

\section{Les Hautes Plaines occidentales durant la période coloniale : déclenchement de la destructuration d'un espace et une société longtemps en équilibre.}

3 Après l'indépendance, l'espace algérien était en pleine mutation; toutefois, toutes les régions n'ont pas évolué au même rythme. Ainsi, malgré les efforts consentis par l'Etat, les Hautes Plaines occidentales restent toujours en marge du développement. Par son immensité et son ouverture, la forte discontinuité de ses pâturages, combinés à un climat rigoureux (un hiver très froid et un été chaud et sec), la steppe a favorisé un mode de vie bien particulier basé sur le nomadisme. Cela explique d'ailleurs l'absence de ville dans la région dans la mesure où l'organisation économique reposait sur des déplacements saisonniers des nomades (achaba), les échanges se faisant avec le Tell en été, et avec le Sahara en hiver. L'autorité politique émanant de la tribu elle-même, l'administration ne pouvait être sédentaire. De cette forme d'adaptation de l'homme avec son milieu naturel, a découlé un certain équilibre écologique, permettant à l'homme de subsister sans détériorer sensiblement son environnement.

Durant l'occupation française, cet équilibre a été rompu, et l'organisation de l'espace steppique s'est modifiée radicalement, avec la mise en place d'une organisation urbaine. La politique coloniale visant à contrôler, à encadrer et à codifier tous les aspects de la vie nomade, a commencé par tracer des limites administratives sur cet espace ouvert. Elle a créé ensuite des centres urbains. Outils essentiels de l'exploitation coloniale, ces derniers répondaient à trois préoccupations principales : le contrôle des populations, le relais du trafic de bétail et le lieu d'asile pour les nomades ruinés. Par ce quadrillage de l'espace 
nomade, la société et l'économie pastorale ont été complètement déstructurés provoquant le démantèlement et la division des tribus, la réduction des parcours et le blocage de l' achaba.

\section{Les deux premières décennies de l'indépendance : la stagnation d'une région.}

5 Après l'indépendance, la colonisation a légué une région en crise et sous-développée. Avec l'occupation de la steppe, le phénomène de regroupement de la population a été déclenché et par conséquent, celui de la sédentarisation des nomades, qui se poursuit jusqu'à nos jours. Depuis, l'Etat représente le principal acteur dans le renforcement du phénomène de regroupement de la population steppique et ce, en utilisant différentes opérations. Cette stratégie a été adoptée au niveau national, afin d'assurer une desserte plus au moins correcte des populations, grâce à la mise en place de différents services sociaux, organisés en un réseau de centres bien hiérarchisé qui encadre tout l'espace national. Cette politique "d'aménagement du territoire », basée sur le développement des villes moyennes et la diffusion des petites villes n'a pas profondément touché la steppe occidentale durant les deux premières décennies de l'indépendance lorsque les Hautes Plaines occidentales appartenaient administrativement à la wilaya de Saïda (Cf. figure. 2). Durant cette période, elle n'a pas été touchée par le processus de développement qu'ont connu les régions du Tell, puisqu'elle comptait tous les ingrédients du sous-développement, taux d'occupation faible, taux de chômage élevé, souséquipement flagrant...

6 C'est la raison pour laquelle elle a été considérée comme l'une des régions les plus déshéritées du territoire national, qu'il fallait soutenir avec d'autres programmes économiques. Elle a bénéficié alors d'un Programme spécial de wilaya lancé à partir de 1966, dont l'objectif était de hisser ces régions marginalisées héritées de la période coloniale, à un certain niveau de développement, en leur donnant les conditions et les moyens nécessaires, pour faciliter leur insertion dans les plans nationaux de développement. 
Figure 2. Evolution des limites administratives dans l'espace des Hautes Plaines occidentales.

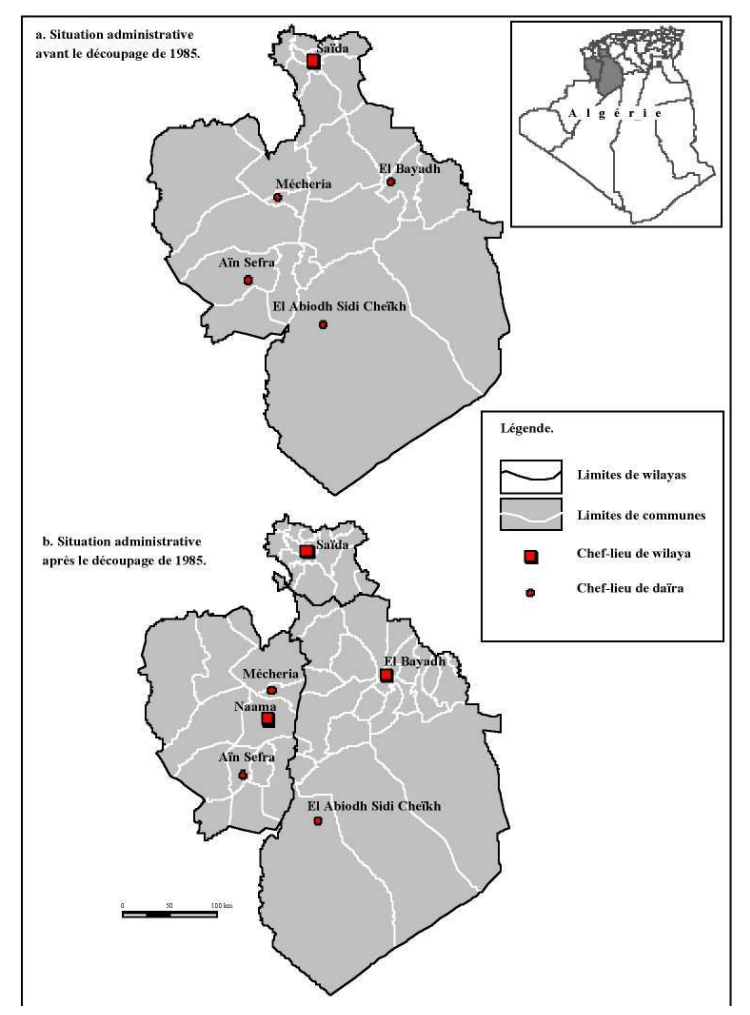

\subsection{Conditions d'intervention du Programme spécial de Wilaya} (P.S.W.)

7 Les caractéristiques socio-économiques qui ont permis à la wilaya de Saïda d'obtenir un Programme spécial (P.S.W.) à partir de 1972 sont les suivantes :

\subsubsection{Un profil socio-économique qui caractérise le sous-développement d'une région.}

8 Le caractère rural de la région est reflété par la structure socio-économique de la wilaya de Saïda, dominée par le secteur agricole qui rassemblait plus des 3/4 du total des actifs d'après le recensement de 1966, taux largement supérieur à la moyenne nationale (56.7\%) (tableau 1). Cependant, l'importance des actifs dans le secteur agricole ne signifie pas que ce dernier était dynamique, car le tiers des actifs agricoles étaient sans travail. D'autre part, " sur les 5.421.904 ha que comptait la wilaya en 1966, la surface agricole utile (S.A.U) ne représentait que 3,3\%, soit une superficie approximative de 179.390 ha, dont $57 \%$ sont occupées par les céréales, avec des rendements faibles, qui varient entre 7 et 8 quintaux à l'hectare; les cultures maraîchères qui se concentrent dans la zone Saïda-Rebahia, n'occupent q'une superficie de 1.556 ha, produisant annuellement 90.000 Qx ; l'arboriculture ne s'éloigne pas plus de cette zone, puisque $80 \%$ des oliviers -à titre d'exemple-se trouvent aux alentours de Rebahia(chef-lieu de la commune de Ouled Khaled, à $4 \mathrm{Km}$ au nord de la ville de Saïda), quant à la steppe, sa seule devise était le bétail, dont la taille était estimée à près de 2 millions de têtes » (Bounouara, 1981). 
Tableau 1. Profil socio-économique de la wilaya de Saïda en 1966.

\begin{tabular}{|l|l|l|l|l|l|l|l|l|}
\hline B.A.E & \multicolumn{2}{|l|}{ Agriculture } & \multicolumn{2}{l|}{ Industrie } & \multicolumn{2}{l|}{ B.T.P } & \multicolumn{2}{l|}{ Tertiaire } \\
\hline Daïras & Effectif & $\%$ & Effectif & $\%$ & Effectif & $\%$ & Effectif & $\%$ \\
\hline Daïra de Saïda & 19654 & 77,8 & 922 & 3,6 & 532 & 2,1 & 4151 & 16,4 \\
\hline Daïras steppiques & 19631 & 77,6 & 749 & 2,9 & 1332 & 5,3 & 3572 & 14,1 \\
\hline Total Wilaya & 39285 & 77,7 & 1671 & 3,3 & 1864 & 3,7 & 7723 & 15,3 \\
\hline Algérie $^{1}$ & 1293413 & 56.7 & 184872 & 8,1 & 128702 & 5,6 & 673985 & 29,5 \\
\hline
\end{tabular}

Source: O.N.S, wilaya de Saïda, série 1, volume 11, 1970.

Quant au secteur secondaire (industrie et BTP), moteur de la croissance économique, il n'occupait que $7 \%$ de l'emploi. Représenté par quelques entreprises nationales (eaux minérales, industries alimentaires...), ce secteur modéré se localisait dans sa totalité dans le nord de la wilaya, la steppe était ainsi dépourvue de toute industrie.

\subsubsection{Un sous-équipement marqué en matière de services éducatifs et sanitaires.}

$10 \mathrm{Au}$ lendemain de l'indépendance, la scolarisation dans la wilaya de Saïda connaissait un retard flagrant, par rapport aux autres wilayas du pays, la steppe était la plus touchée par ce phénomène, puisqu'en 1966, elle possédait le taux de scolarisation le plus faible du territoire national. Cette situation a permis à l'analphabétisme de régner dans la région, puisque d'après les données du recensement, $85 \%$ des personnes de plus de 10 ans étaient analphabètes, contre $74.7 \%$ au niveau national. Jusqu'en 1971, le progrès de la scolarisation était encore nettement insuffisant, les taux de scolarisation étaient toujours faibles, 55 \% à Aïn Sefra, 50 \% à Mécheria et $35 \%$ à El Bayadh.

11 Le manque d'équipement et d'encadrement a marqué aussi le secteur sanitaire. Les trois hôpitaux qui existaient dans la wilaya étaient tous vétustes et dataient du début du siècle: Saïda (222 lits), El Bayadh (112 lits) et Aïn Sefra (40 lits). De plus, la wilaya toute entière ne comptait que 25 médecins en 1966, soit un praticien pour 12000 habitants (moyenne nationale : 1 médecin pour 8300 habitants), et un infirmier pour 4200 habitants.

\subsubsection{Un sous-développement de la région qui s'est répercuté sur ses villes.}

Le sous-équipement marqué de la wilaya et l'insuffisance de ses structures économiques pouvant permettre le lancement du développement de la région, a touché profondément les centres urbains de la wilaya et notamment le chef-lieu de wilaya Saïda. En fait, Les répercussions de ce sous-équipement de la wilaya sont d'ordre social, suite à une constitution d'une masse considérable de sous-employés ou de chômeurs. La ville de Saïda par exemple, possédait un des taux de chômage les plus élevé de toute l'Algérie avec $40 \%$ en 1966, alors que la moyenne nationale n'atteignait même pas les 35\% (tableau 2). 
Tableau 2: Taux de chômage dans les villes de la wilaya de Saïda (1966).

\begin{tabular}{|l|l|l|l|}
\hline & Population active & STR1 \& STR2 & Taux de Chômage \\
\hline \hline Ville de Saïda & 7363 & 2937 & 39,9 \\
\hline Villes steppiques & 6593 & 2108 & 32,0 \\
\hline
\end{tabular}

SOURCE: O.N.S, WILAYA DE SAÏDA, SÉRIE 1, VOLUME 11, 1970.

13 Ce taux était en fait gonflé par un afflux considérable de populations, venant essentiellement des zones rurales et steppiques, totalement déshéritées après l'indépendance. Les répercussions d'ordre spatial sont liées à la prolifération des bidonvilles. En effet, la ville de Mécheria qui ne possédait pas d'habitat précaire dans son tissu urbain en 1966, a compté 460 véritables gourbis en 1976; le même phénomène se retrouve à Aïn Sefra, qui a connu l'installation de 126 gourbis à Mouilah (agglomération secondaire de la commune de Aïn Sefra (1987)). Mais c'est à El Bayadh que ce phénomène était le plus important, car des quartiers entiers d'habitat précaire se sont développés autour de la ville, du ksar de Belkheïra au nord, à celui de Hadj Bahous à l'est, aux abattoirs à l'ouest et sur le versant sud.«L'estimation du nombre de gourbis était un peu exagérée, dans la mesure où a été donné un chiffre de 3000 constructions précaires, abritant près de 20000 habitants durant la période 1966/1977, alors que la ville ne s'est accru que de près de 13 000 habitants durant cette décennie, et sur la base d'un croit démographique de 3,2 \%, l'apport migratoire peut être estimé à 6700 personnes ou migrants » (Couderc, 1979).

14 Toutes ces conditions qui caractérisaient la wilaya de Saïda, ont permis de la classer comme l'une des régions les plus déshéritées du pays ; il était donc temps de doter cette région d'un programme spécial, afin de lui permettre d'atténuer le retard qu'elle connaissait en matière d'équipements productifs et sociaux.

\subsection{L'intervention étatique à travers les plans nationaux et le Programme spécial de wilaya.}

15 La situation économique et sociale critique de la wilaya de Saïda, et notamment de sa partie steppique après la première décennie de l'indépendance, s'est accentuée après le « total oubli » qu'elle a connu durant le premier plan triennal (1967-1969). Les structures politiques et économiques étaient certes en cours de mise en place à cette période, mais la wilaya n'avait presque rien reçu, à part quelques actions destinées à limiter le chômage (programme de plein emploi, développement économique rural).

16 Le premier plan quadriennal (1970-1973) n'allait pas non plus permettre de satisfaire les besoins et les aspirations de la population. Ainsi, la wilaya de Saïda, une des vastes régions du pays (4.5\% du territoire national), n'a reçu que 186 millions de dinars, soit 0,55 \% de l'ensemble des investissements projetés sur le territoire national. Dans ces conditions, la situation du sous-développement risquait de s'accentuer par rapport aux autres wilayas et notamment celle du Tell. A cheval sur le premier et le deuxième plan quadriennal et mis en œuvre pour une durée de cinq ans (1972-1977), le Programme spécial de wilaya (P.S.W) intervenait donc à temps, avec un investissement total de près d'un milliard de dinars. La répartition sectorielle des investissements était judicieuse dans la mesure où les $3 / 4$ de la 
somme totale accordée par le P.S.W, étaient destinés aux secteurs productifs, avec une priorité donnée à l'industrie, l'hydraulique, l'agriculture et la mise en valeur agricole, pour une valeur de 750 millions de dinars. L'industrie, alors considérée comme le secteur le plus apte à redynamiser cet espace, par les emplois créés, les revenus distribués, les produits fabriqués et distribués, ainsi que par les relations et les échanges inter-urbains qu'elle crée, apparaissait la plus apte à permettre l'intégration de cette région marginalisée à l'espace national. Elle a d'ailleurs reçu 450 millions de dinars, le reste du financement étant destiné à l'hydraulique, à l'agriculture et à la mise en valeur agricole. Cette répartition des investissements était peut-être rationnelle, et imposée par le contexte socio-économique qui marquait la wilaya toute entière, mais le vrai problème, résidait dans la distribution géographique de ces investissements. Le nord de la wilaya de Saïda (daïra de Saïda) paraissait plus favorisé par rapport aux daïras steppiques, en absorbant plus de 78,3\% des investissements productifs alors qu'elle ne représentait même pas $42 \%$ de la population totale de la wilaya (tableau 3).

Tableau 3 : Répartition sectorielle et géographique des investissements productifs du P.S.W. de Saïda (en millions de dinars).

\begin{tabular}{|l|l|l|l|l|l|l|}
\hline & \multicolumn{2}{|l|}{ Daïra de Saïda } & \multicolumn{2}{l|}{ Daïras steppiques } & \multicolumn{2}{|l|}{ Total wilaya } \\
\hline \hline Secteurs & Valeur & $\%$ & Valeur & $\%$ & Valeur & $\%$ \\
\hline \hline Industrie & 450 & 100 & - & - & 450 & 60,0 \\
\hline Agriculture & 70 & 60,9 & 45 & 39,1 & 115 & 15,3 \\
\hline Hydraulique et mise en valeur & 67 & 36,2 & 118 & 63,8 & 185 & 24,7 \\
\hline Total & 587 & 78,3 & 163 & 21,7 & 750 & 100,0 \\
\hline
\end{tabular}

SOURCE: HADEID, 1996.

17 Si les daïras steppiques ont obtenu près de $40 \%$ des investissements agricoles, et plus de $60 \%$ de ceux de l'hydraulique et de la mise en valeur, 83 millions de dinars étaient destinés au projet de Aïn Skhouna, pour la création d'un périmètre irrigué à Daïet Zraguet. Et si sur le plan physique, la zone de Aïn Skhouna appartient au domaine steppique ou prés-steppique, sur le plan administratif, elle appartient à la daïra de Saïda. Ainsi, les daïras steppiques dont la superficie dépasse les $93 \%$ de l'ensemble de l'espace de la wilaya, n'ont finalement touché que 35 millions de dinars (sur les 185 millions, soit 19\% ) qui ont été employés à la recherche des forages et à l'entretien des puits d'eaux en zone steppique.

18 On parlait alors d'une multitude de petits projets industriels : menuiserie et briqueterie à Aïn Sefra, unité de montage de radiateurs à Mécheria et unité de montage de moteurs électriques à El Bayadh. Mais aucun de ces projets n'a vu le jour, à part trois unités artisanales de tissage des tapis, employant moins de 20 travailleurs chacune, et installées dans les trois chefs-lieux de daïras. En réalité, la daïra de Saïda s'est accaparée la totalité des projets industriels, qui étaient d'une importance telle que les crédits débloqués par le P.S.W. étant insuffisants, il a été nécessaire de solliciter le premier et le deuxième plan quadriennaux pour compléter le financement industriel. 
19 De plus, la répartition des investissements du premier et du deuxième plans quadriennaux étaient aussi inégale que celle du P.S.W. : les daïras steppiques n'ont reçu que $15 \%$ des investissements agricoles pour le premier plan et $50 \%$ pour le deuxième, et seulement $25 \%$ de ceux destinés à l'hydraulique. Ainsi l'écart s'est creusé entre la steppe et la partie nord de la wilaya de Saïda qui s'était découverte un avenir industriel. La steppe a donc été quelque peu délaissée, malgré les crédits importants de la Révolution agraire, "dont la part prépondérante des investissements était destinée durant la troisième phase, à la création des villages pastoraux» (Couderc, 1979).

\section{Les Hautes Plaines occidentales après 1985. Une relance économique médiocre, mais un début d'encadrement de la population steppique en matière d'équipements et de services.}

Après l'indépendance, dans un souci d'homogénéisation et d'encadrement de tous les espaces du pays, l'Etat a engagé plusieurs découpages administratifs. Les deux premiers (1963 et 1975), n'ont pas profondément touché notre région des Hautes Plaines occidentales, le nombre de ses communes n'a pratiquement pas changé durant plus de 20 ans. Ce n'est qu'à l'occasion du dernier découpage de 1985 (figure 2), que la région a enfin subi un changement radical de son organisation administrative. En effet, d'un côté, le nombre de communes a plus que doublé et d'un autre, la région a été scindée en deux wilayas, celle d'El Bayadh et de Naama. Ce dernier découpage a donc permis à la région de se détacher de la wilaya de Saïda qui s'est, à l'occasion, "débarrassée" d'une vaste région qui était difficile à gérer et à équiper.

21 Par la création de ces deux wilayas, cette refonte administrative représente un acquis sans précédent pour la population steppique. Elle devait théoriquement permettre la réalisation des objectifs de l'Option Hauts Plateaux (O.H.P), qui visait l'utilisation accélérée et prioritaire du potentiel humain et économique, tout en prenant en considération les spécificités locales (société nomade, espaces vastes et contraignants...). Cet objectif s'insère également dans la future politique d'aménagement du territoire, avec son triple aspect, d'élimination des disparités régionales, de mise en valeur et de sauvegarde des ressources naturelles, et d'harmonisation du peuplement. Voyons à présent les efforts consentis par l'Etat après cette refonte administrative des Hautes Plaines occidentales.

\subsection{Un recul du sous-emploi après 1985.}

L'étude de l'évolution du taux de chômage est un bon indicateur pour l'analyse socioéconomique d'une région. L'approche globale montre nettement d'après le tableau 4 , la baisse qu'a connue le taux de chômage entre 1966 et 1987. Mais le plus remarquable est la baisse de ce taux entre 1966 et 1977, car il passe de $26,5 \%$ à $11,1 \%$. Cette période correspond au premier et au deuxième plan quadriennal ainsi qu'au P.S.W. Ces programmes sont-ils bien à l'origine de la création d'un nombre si important d'emplois, permettant ainsi cette résorption du chômage? 
Tableau 4 : Evolution du taux de chômage dans les Hautes Plaines occidentales.

\begin{tabular}{|l|l|l|l|l|}
\hline & 1966 & 1977 & 1987 & 1998 \\
\hline \hline STR & 9.727 & 4.411 & 14.072 & 25.145 \\
\hline Population active & 36.716 & 39.664 & 78.508 & 130.416 \\
\hline Taux de chômage (\%) & 26,5 & 11,1 & 17,9 & 19,3 \\
\hline Taux de chômage en Algérie (\%) & $35 \%$ & 22 & 21.4 & 27.6 \\
\hline
\end{tabular}

SOURCE: O.N.S., 1966, 1977, 1987, 1998.

Durant la deuxième décennie, le taux de chômage a augmenté pour atteindre 17,9 \%. La croissance démographique d'une part, et l'achèvement de plusieurs projets entrepris par le secteur des B.T.P, représentent probablement la vraie cause de cette remontée. En raison de la crise économique et politique du pays durant les années quatre vingt dix, le chômage devrait théoriquement augmenter comme ce fut le cas en 1966. Les données du recensement de 1998 indiquent un taux de chômage de l'ordre de 19.3\%, relativement élevé par rapport à celui de 1987. En réalité, le sous-emploi est absorbé beaucoup plus par l'emploi dans le secteur tertiaire et dans le secteur informel. Ce phénomène induit par l'arrêt des investissements productifs (industriels surtout) depuis les années quatre vingt, n'est pas un fait exclusif dans la steppe, mais il touche l'ensemble du territoire national. A l'avenir, la tension s'exercera certainement sur les centres urbains qui connaissent des taux de croissance élevés, alimentés en grande partie par la zone éparse et la sédentarisation des nomades.

\subsection{Des efforts économiques insuffisants.}

Durant cette période, les efforts économiques de l'Etat n'ont pas beaucoup favorisé le secteur industriel, dans la mesure où la politique d'industrialisation n'a pas touché la steppe, alors que le nord de la wilaya de Saïda, a été totalement transformé après la création de deux zones industrielles dans la région et de la papeterie de l'ex-SONIC. Les premières unités industrielles n'ont été implantées dans la région qu'après 1978, et encore de façon ponctuelle et isolée. El Bayadh a été la première à être touchée par ce processus, avant même de devenir chef-lieu de wilaya. Mais, malgré les quelques unités industrielles implantées à Ras $\mathrm{El} \mathrm{Ma}$, Mécheria et Bougtob, l'industrie moteur de la croissance économique, est loin d'être une activité de base dans la steppe occidentale. Cette région contient par contre, assez de matières premières pour généraliser les petites et moyennes industries, telles que la fabrication des matériaux de construction, les carrières, le tissage... En réalité, la faiblesse du secteur secondaire dans la région s'explique largement par l'absence des conditions nécessaires à cette activité (éloignement des grands centres urbains et des ports, manque d'eau et d'énergie, milieu physique contraignant...) (Hadeid, 1996).

Ainsi, la majorité des investissements des différents programmes planifiés a touché les autres secteurs : en premier lieu le secteur primaire qui s'est accaparé la totalité des 
subventions octroyées par les programmes sectoriels. L'hydraulique absorbe presque la moitié des investissements $(49,4 \%)$, à cause de son importance dans un milieu où l'eau est un élément rare, notamment pour l'élevage. L'autre moitié a concerné essentiellement, le développement de l'agriculture, avec des opérations telles que les améliorations foncières, la création d'unités pastorales et agro-pastorales, le développement de la culture irriguée (dans l'Atlas saharien et sur les marges steppiques essentiellement), les infrastructures rurales et de services (dispensaire vétérinaire, laboratoire vétérinaire, hangars de stockage..), qui ont absorbé plus de $70 \%$ du total des subventions destinées à l'agriculture. Mais, malgré ces efforts, ce secteur dominé par le pastoralisme connaît actuellement une crise qui est loin d'être surmontée. Les aléas climatiques (sécheresse) d'une part, la réduction des transhumances, la sédentarisation du cheptel et les pratiques "sauvages" des gros éleveurs d'autre part, ont conduit à la dégradation du couvert végétal qui ne peut plus supporter la charge actuelle. S'il l'on ajoute à tout cela l'augmentation des prix des aliments du bétail, les petits nomades ou éleveurs risquent bientôt de ne plus pouvoir exercer le pastoralisme. La sédentarisation en ville devient alors incontournable comme le confirme le taux de la population nomade qui, dans la région, a connu une nette diminution entre 1966 et 1998, en passant de $65 \%$ à $21 \%$. (Hadeid, 2006).

\subsection{Un effort non négligeable en matière d'équipements et de services.}

A côté de ses insuffisances économiques, on ne peut nier les efforts de l'Etat en matière d'équipements sociaux en général. En effet, sur le plan éducatif à titre d'exemple, l'Etat a fait beaucoup de progrès dans l'augmentation du taux de scolarisation, ce dernier était l'un des plus bas dans toute l'Algérie en 1966, avec 122 élèves sur 1000 enfants. Ces taux étaient encore très faibles jusqu'en 1971, même dans les agglomérations urbaines: El Bayadh, Mécheria et Aïn Sefra, dont les taux étaient respectivement: 35\%, 50\%, 55\% (Couderc, 1979). A présent, le progrès dans la scolarisation est considérable dans la mesure où il touche même la population nomade. Cet aspect est devenu tellement important pour les nomades, qu'ils ont été obligés de réduire leurs déplacements saisonniers, afin de permettre leurs enfants d'accéder à l'école. Le taux de scolarisation dépasse maintenant le seuil de $80 \%$ pour les garçons et $76 \%$ pour les filles, taux légèrement au dessous de la moyenne nationale ( $83.1 \%$ pour les garçons et $78.3 \%$ pour les filles) d'après le recensement de 1998.

Si l'enseignement primaire est accessible à tous les enfants de la région, le cycle moyen l'est aussi, puisque pratiquement chaque chef-lieu de commune possède un C.E.M. (collège d'enseignement moyen), à l'exception de huit chefs-lieux dans la wilaya d'El Bayadh, et quatre dans la wilaya de Naama. Ces ACL (agglomérations chefs-lieux) sont tous issus du découpage de 1985. Quant à l'enseignement secondaire, il nécessite par contre de longs déplacements, dans la mesure où le nombre de lycées dans la région est assez limité, et où ils se concentrent dans les villes principales et dans quelques bourgs. Toutefois, il faut souligner que la situation actuelle est bien meilleure puisque avant 1975, El Bayadh possédait le seul lycée de la région. 


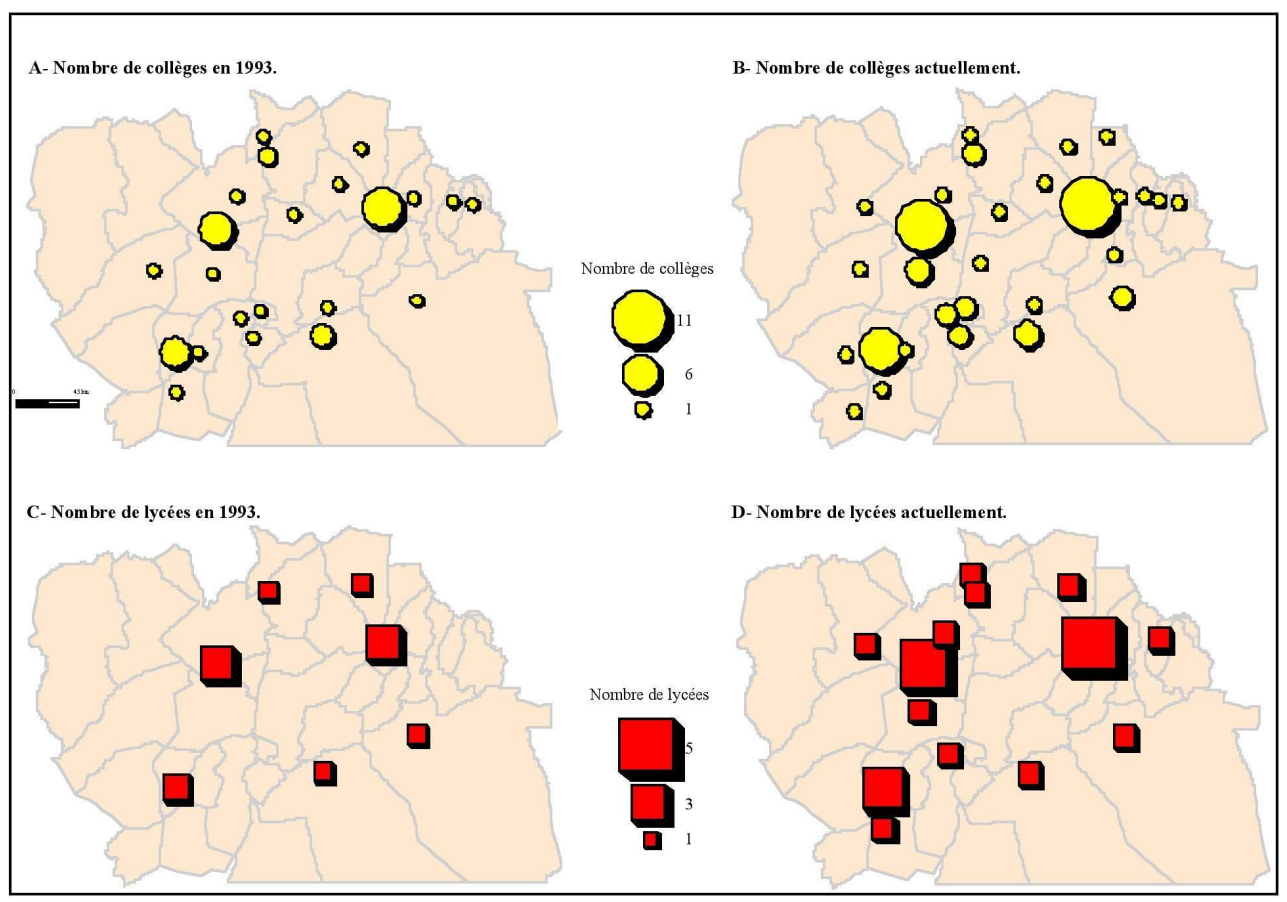

Figure 3. Evolution des établissements de cycle moyen et secondaire dans les wilayas de Naama et d'El Bayadh.

D'autres domaines montrent les efforts de l'Etat en matière d'équipements. Il s'agit de l'électrification notamment en milieu rural, les chiffres obtenus concernent l'espace communal tout entier, mais indiquent néanmoins, le progrès en ce domaine, car l'électrification a touché les espaces les plus enclavés. Il existe toutefois des insuffisances dans quelques communes. En effet, 14 sur les 22 communes de la wilaya d'El Bayadh, ont des taux d'électrification qui dépassent les $50 \%$; les moins équipées sont en général les communes steppiques à vaste superficie, qui demandent beaucoup plus de travaux que les petites communes de l'Atlas saharien par exemple. Les communes urbaines sont en fait les plus équipées (Hadeid, 2002).

Les autres tentatives sont aussi à signaler, par exemple les opérations de désenclavement des agglomérations steppiques, par la création des axes routiers. Les efforts sont considérables si on remarque l'évolution du réseau routier durant toutes ces années, bien qu'elle n'ait pu réellement suivre le rythme de développement d'un nombre élevé d'agglomérations. Ainsi, beaucoup de localités nouvelles sont mal desservies, puisque des chefs-lieux de communes sont liés par des pistes au réseau routier existant, c'est le cas de El Mehara, Krakda... L'immensité des espaces dans la steppe occidentale, rend difficile l'étoffement des voies de communications, mais en dépit de cela, beaucoup de projets sont inscrits dans des programmes sectoriels ou de P.C.D, qui concernent des liaisons routières entre agglomérations. Par exemple, la liaison Naama-Asla sans passer par Aïn Sefra semble, d'après leurs déclarations, avoir satisfait beaucoup de gens. De même, les gens de Asla ou venant de la wilaya d'El Bayadh par Chellala et voulant se rendre à Naama pourront désormais gagner du temps et des kilomètres grâce aux nouveaux aménagements routiers (figure 4). 


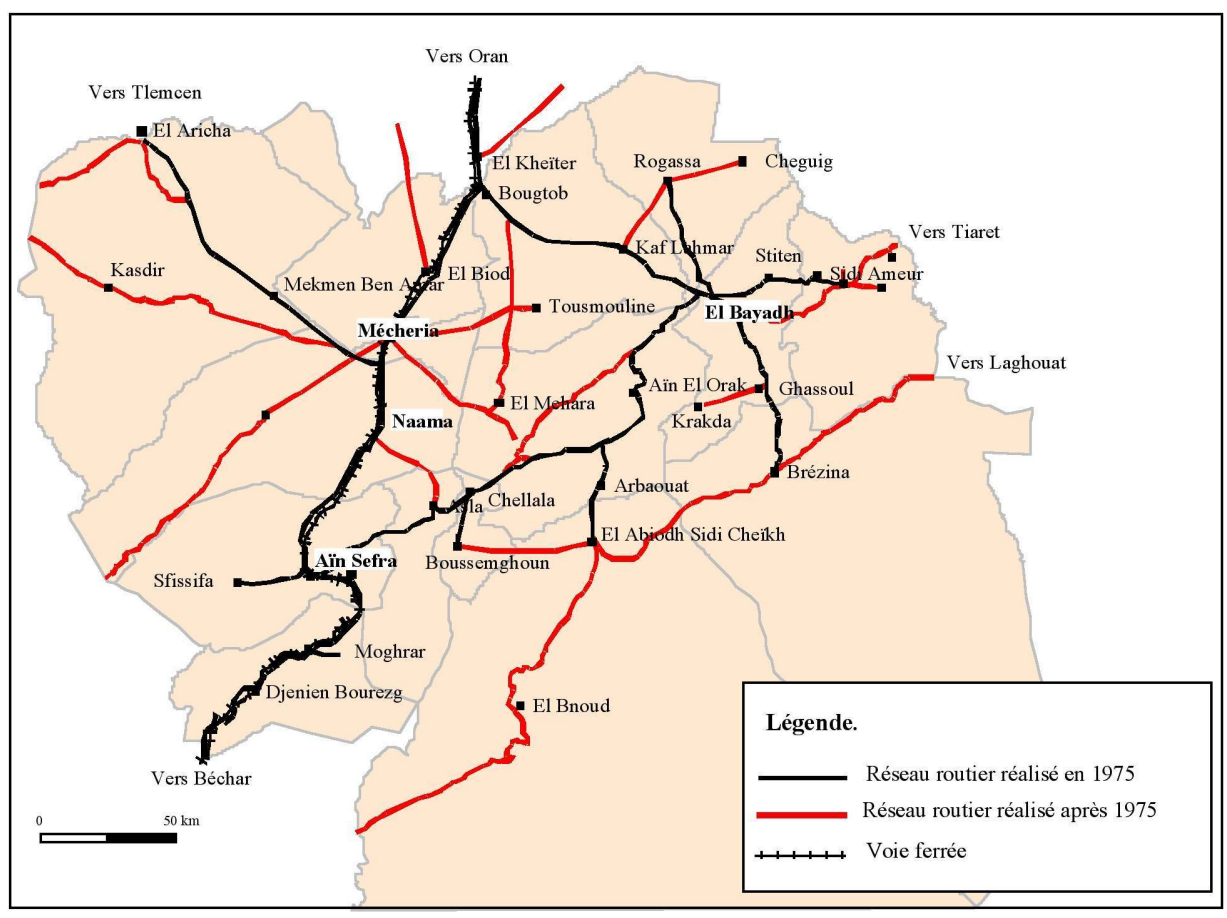

Figure 4. Evolution du réseau routier dans la wilaya de Naama et d'El Bayadh. sanitaires de grande capacité d'accueil (hôpitaux) se situent au niveau des principaux centres urbains : El Bayadh, Mécheria, Aïn Sefra et El Abiodh Sidi Cheïkh. De même, les polycliniques se concentrent sur sept agglomérations seulement pour toute la steppe. Seuls les centres de santé et les salles de soin semblent assez bien répartis sur la région, mais ces derniers ne sont pas en mesure de traiter certains cas nécessitant un déplacement urgent vers un des hôpitaux de la région. Le sous-équipement de la steppe en matière de santé est très marquant. En voyant la carte de la répartition des pharmacies, il est évident que le déplacement vers les centres urbains pour se procurer les médicaments demandés est obligatoire. La concentration des pharmacies est extraordinaire dans la mesure où presque les $3 / 4$ se situent au niveau d'El Bayadh (34.4\%), Mécheria (25\%) et Aïn Sefra (14.1\%).

Quant à l'encadrement, il n'est pas meilleur. La carte de la répartition des médecins généralistes et spécialistes publics ou privés (figure 5) montre de grandes différences. En effet, si les généralistes publics sont assez bien répartis sur la région, il n'est pas de même pour les médecins privés qui préfèrent s'installer dans les grandes agglomérations urbaines. La même remarque est à signaler pour les dentistes. Pour les spécialistes, leur nombre est presque négligeable et se concentrent entièrement autour des trois villes principales de la steppe (El Bayadh, Mécheria et Aïn Sefra). Le rapport entre le nombre d'habitants et le nombre de médecins tous types confondus donne un indice de 1 médecin pour 1073 habitants, en comparaison pour la même période, l'indice au niveau national est de l'ordre d'un médecin pour 984 habitants. Autrement dit, la steppe n'est pas très loin de la moyenne nationale; cependant, sur le plan spatial, il existe des différences énormes. Dans 29 communes sur 34 le rapport médecin-population est au-dessous de la moyenne nationale. Seules, cinq communes ont un médecin pour moins de 1000 habitants. Là aussi, on relève une opposition entre communes ksouriennes moins défavorisées et les communes steppiques proprement dites très sous-équipées. 


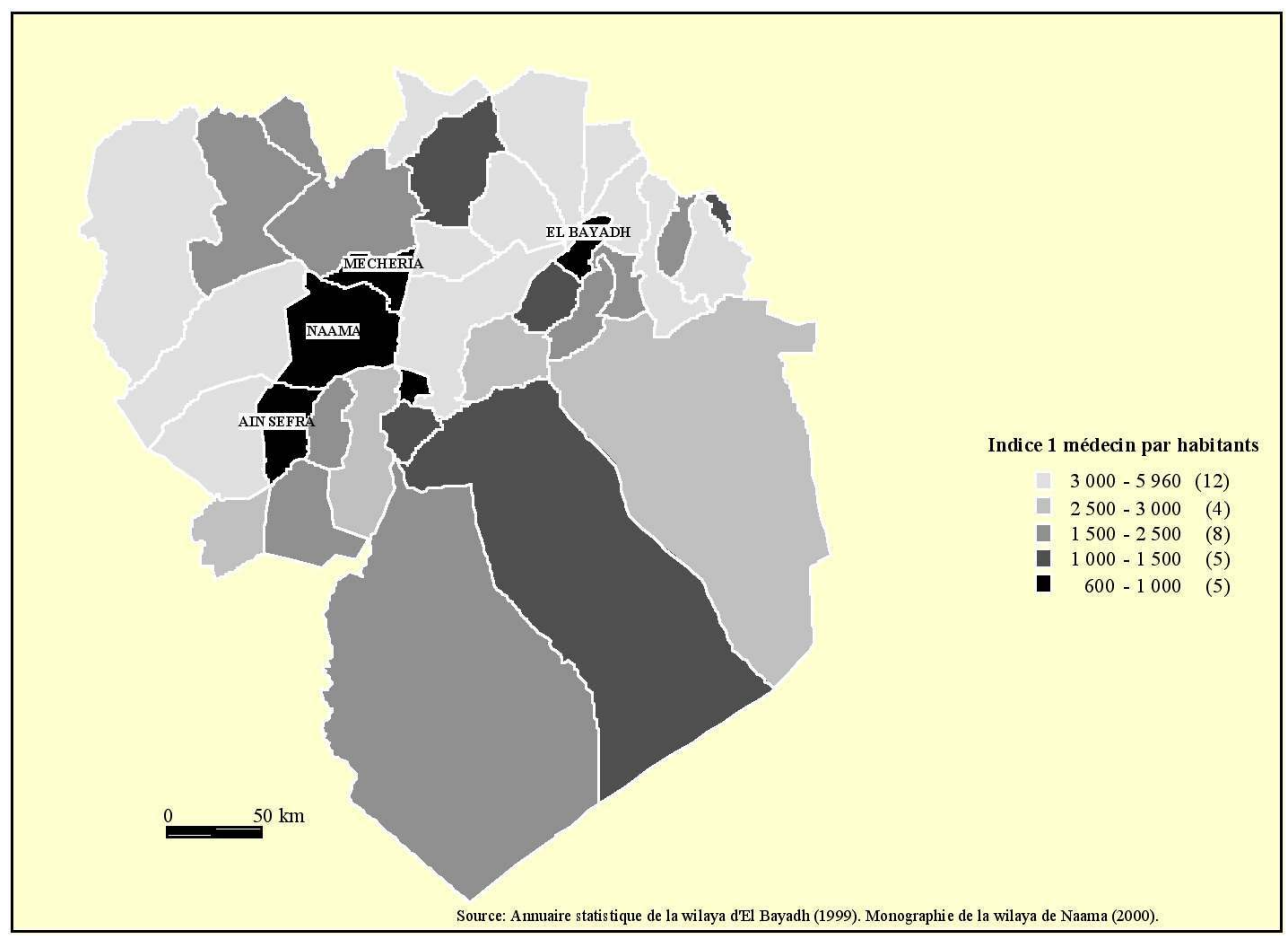

Dans les autres secteurs, on constate le même phénomène de concentration des équipements au niveau des agglomérations urbaines par opposition au sous-équipement des zones rurales. C'est le cas des infrastructures sportives et culturelles. Seuls, les équipements de culte sont en abondance et assez bien répartis sur la steppe.

La répartition des établissements postaux est certes bien diffusée et chaque commune possède le sien, mais le nombre d'abonnés au téléphone ne suit pas. Là aussi, la concentration est urbaine : La ville d'El Bayadh à elle seule rassemble presque le tiers des abonnés pour toute la région. Si on ajoute Mécheria et Aïn Sefra, ce taux est de l'ordre de $66.2 \%$ et si on inclut les autres agglomérations urbaines, nous constatons que $82 \%$ des abonnés s'agglutinent autour de six centres urbains, alors qu'ils concentrent moins de $75 \%$ de la population totale.

Les efforts de l'Etat apparaissent donc conséquents sur le plan quantitatif et qualitatif, mais la répartition spatiale des équipements reste peu satisfaisante car leur regroupement dans les trois ou quatre principales villes de la steppe ne rend pas service aux habitants de la région. Les investissements dépensés ne sont pas négligeables d'autant plus qu'ils sont diversifiés, mais ils n'obéissent pas réellement à une logique spatiale tant souhaitée par les populations rurales.

\section{Les Hautes Plaines occidentales aujourd'hui et demain.}

Durant cette période qui a bouleversé non seulement l'espace steppique, mais tout le pays, l'avenir de la steppe apparait incertain ; certes beaucoup de projets, de 
programmes, d'investissements sont versés dans la steppe, mais cela est-il suffisant pour sortir la steppe de son enclavement, de son sous-développement?

Les efforts d'équipement sont certes impressionnants, mais les projets productifs restent en définitive insuffisants. Si ses retombées sur l'emploi sont conséquentes, elles concernent un emploi non productif si on exclut le secteur de l'agriculture appuyé par la politique de la mise en valeur agricole, dans une étape où la steppe est en train de " perdre » sa vocation pastorale. En effet, depuis la promulgation de la loi de 1983 pour l'accession à la propriété foncière agricole, la politique de mise en valeur agricole lancé par l'Etat algérien en vue de redynamiser l'agriculture algérienne se généralise. A présent, les périmètres de mise en valeur sont répartis sur l'ensemble de l'espace steppique. Certes, la surface totale de ces périmètres demeure négligeable par rapport à la superficie totale, mais il faut noter que les actifs agricoles issus de cette opération représentent presque le tiers du total des actifs du secteur primaire. Cette tendance se confirme puisque le PNDA lancé à partir de 2000 a redonné une impulsion à cette politique de mise en valeur agricole. Ce plan ambitieux permet la subvention de toute une série d'opérations liées à l'équipement des exploitations de mise en valeur (construction de bassin de stockage d'eau, installation de système goutte à goutte, creusement de puits...) (Hadeid, 2006).

De même, il faut soulever le point lié à la diversité des sources d'investissements durant cette période. En effet, hors le budget alloué à la wilaya, cet espace a bénéficié d'autres sources financières supplémentaires : budget de la relance économique (2001), fonds du Sud (2001) et dernièrement le fonds de la Steppe (2002).

Il est très difficile actuellement d'obtenir des données concernant la répartition sectorielle et financière des projets selon les différentes sources : budget de la relance, fonds du Sud... Notre investigation sur terrain, nous a permis quand même d'obtenir quelques données qui, à défaut d'être exhaustives, peuvent donner une idée de la répartition des investissements au niveau de la wilaya. Ces données ont été recueillies auprès de la Direction de la planification et de l'aménagement du territoire (DPAT). Elles concernent uniquement la wilaya d'El Bayadh pour l'année 2001. (Tableau 8)

Tableau 8 : Répartition des investissements par source dans la wilaya

d'El Bayadh en 2001.

\begin{tabular}{|l|l|l|}
\hline SECTEURS & $\begin{array}{l}\text { Montant } \\
\text { (en millier de dinars) }\end{array}$ & $\%$ \\
\hline \hline Sectoriel & $1.848 .329,4$ & 31,0 \\
\hline Programme de relance & 2.523 .815 & 42,4 \\
\hline Fonds du Sud & 1.582 .000 & 26,6 \\
\hline Total & $5.954 .144,4$ & 100,0 \\
\hline
\end{tabular}

SOURCE : DPAT D'EL BAYADH, 2001. 
décennie. Que se soit dans le domaine de l'agriculture, de l'hydraulique ou en matière
d'infrastructures économiques et administratives, la wilaya d'El Bayadh a bénéficié d'une dizaine de projets s'inscrivant dans plusieurs opérations de financement. Le seul inconvénient de ces opérations est celui de leur répartition sectorielle et géographique. Sur le plan sectoriel, la ventilation des investissements selon les sources montre l'absence de coordination entre les différents programmes. Par exemple, entre le programme sectoriel et le Fonds du Sud, la répartition par secteur est presque la même ; autrement dit, le secteur de l'agriculture et l'hydraulique ne dépassent pas les $10 \%$ du total des investissements pour les deux programmes. Dans une région à vocation pastorale comme celle de la steppe, les investissements destinés à ce secteur apparaissent donc négligeables. Le secteur non agricole s'accapare partout la majorité des investissements (plus de 90\%). Cette répartition inégale des investissements n'est pas en mesure de régler les problèmes accrus que connaît la steppe, mais au contraire, elle contribue à creuser l'écart en matière d'équipement (tableau 9).

Tableau 9 : Répartition des investissements par source dans la wilaya

d'El Bayadh en 2001 (en millier de dinars).

\begin{tabular}{|c|c|c|c|c|}
\hline \multirow{2}{*}{ SECTEURS } & Sectoriel & $\begin{array}{ll}\text { Fonds } & \mathrm{du} \\
\text { Sud } & \end{array}$ & $\mathrm{du}$ & \\
\hline & Valeur * $\%$ & Valeur* & $\%$ & \\
\hline Agriculture / hydraulique & $183.829,4$ & 9,9 & 145.000 & 9,2 \\
\hline Autres & 1.664 .500 & 90,1 & 1.437 .000 & 90,8 \\
\hline Total & $1.848 .329,4$ & 100,0 & 1.582 .000 & 100,0 \\
\hline
\end{tabular}

SOURCE : DPAT D'EL BAYADH, 2001.

41 Sur le plan géographique, la répartition des investissements reste insuffisamment organisée. Certes, elle obéit à une priorité liée à la taille de la population, mais cela n'est pas suffisant dans la mesure où les centres urbains les plus importants concentrent déjà la majorité des équipements et des infrastructures au détriment de l'arrière-pays.

De même, l'analyse de l'aide octroyé aux deux wilayas de Naama et d'El Bayadh suite à la dernière visite du Président de la république 2003, montre que plusieurs secteurs ont été concernés. L'habitat, l'énergie, l'hydraulique, la santé et les travaux publics représentent les secteurs les mieux dotés en investissements, leur ventilation ayant été appliquée en 
fonction de la demande des autorités locales. Mais là encore, le pastoralisme ne figure pas dans la liste des secteurs économiques concernés. L'agriculture n'a représenté que 7\% des investissements pour la wilaya d'El Bayadh et $4 \%$ pour Naama. Sur le plan spatial, les disparités sont également confirmées : dans la wilaya d'El Bayadh, le chef-lieu de wilaya domine avec cinq projets, suivi par Bougtob et seulement trois pour El Abiodh Sidi Cheïkh. Il faut toutefois souligner que, dans cette wilaya, la répartition des projets n'est pas monopolisée par les grands centres urbains qui n'ont obtenu que $40 \%$ du nombre total des projets. Dans la wilaya d'El Bayadh, près de $60 \%$ des communes ont été touchées alors que $75 \%$ l'ont été dans la wilaya de Naama. En revanche, dans la wilaya de Naama, le cheflieu de wilaya, Mécheria et Aïn Sefra se sont accaparés plus de 70\% des projets (figure 6).

Figure 6 Nombre de projets octroyés par commune lors de la visite du Président de la république en 2003.

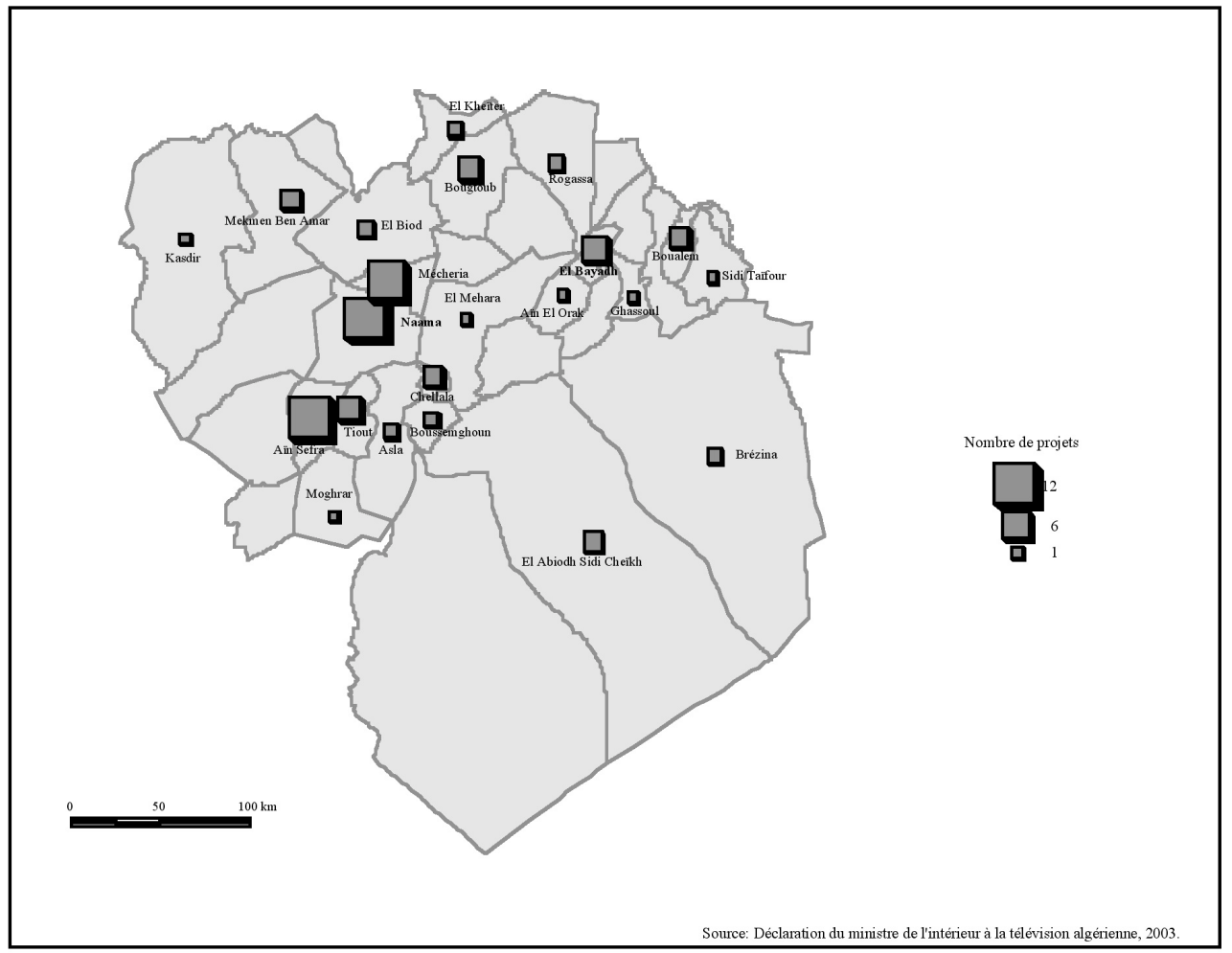

Cette répartition inégale à la fois sur le plan sectoriel et géographique s'est en fait calquée sur les programmes et financements mis en œuvre jusqu'alors, démontrant ainsi que la politique de développement dans notre pays n'est pas suffisamment réfléchie sur le plan spatial, engendrant des disparités locales assez conséquentes en matière de développement et d'équipement.

Les différents programmes et plans nationaux entrepris par l'Etat depuis l'indépendance jusqu'à présent, ont suivi un système de développement sectoriel dépourvu d'une réelle vision géographique inscrite dans une stratégie territoriale plus globale où tous les aspects du développement auraient été traités. Par conséquent, les actions entreprises par l'Etat dans la steppe n'ont finalement conduit qu'à accroître les déséquilibres amorcés durant la période coloniale. Désormais, le problème de la steppe n'est pas seulement lié au chômage, au sous-équipement ou au désenclavement mais, de manière encore plus préoccupante à sa dégradation et à sa désertification. Ce phénomène écologique et dont 
les causes sont d'ordre anthropiques, ne cesse de s'étendre et menace de compromettre toute action de développement future. (Hadeid, 2008)

Dans une période caractérisée par un « désengagement » de l'Etat, il ne faut pas attendre de l'Etat la solution de tous les problèmes. Les actions d'une nouvelle politique d'aménagement du territoire, et notamment dans un espace où l'aspect communautaire est très présent, doivent obligatoirement s'inscrire dans la concertation avec les différents partenaires qui réclament et nécessitent un consensus quant à sa réalisation.

\section{Conclusion.}

L'espace algérien tel que l'on voit actuellement est la combinaison de plusieurs modes d'occupation de l'espace, qui se sont succédés à cours de son histoire. A l'organisation spatiale de la période précoloniale, basée sur des solidarités sociales et des complémentarités spatiales, est venue se plaquer une organisation différente qui en a bouleversé les fondements. Après l'indépendance, l'Algérie n'avait pas d'autre choix que de redémarrer son économie sur l'assise territoriale héritée de la période coloniale tout en essayant d'injecter des programmes de développement vers l'intérieur du pays et ce, en vue d'assurer un certain équilibre spatial et social. La politique d'aménagement du territoire se limitait ainsi, à cette volonté de rééquilibrage spatial d'un pays aussi vaste que l'Algérie. En réalité, la politique d'aménagement du territoire ne représentait pas une priorité de l'Etat dans la mesure où le contexte institutionnel et les procédures de planification étaient fortement marqués par la décision centrale. Autant, la mission de l'aménagement du territoire se rattachait successivement à plusieurs autorités (Ministère de la planification, Ministère de l'habitat,...), autant la vision sectorielle dominait largement sans grand souci de cohérence par rapport aux réalités locales et au détriment du volet spatial. Enfin, l'absence d'un débat public et de concertation a été derrière la marginalisation des expressions et des spécificités locales.

Les Hautes Plaines steppiques occidentales représentent l'une des régions du pays qui sont "victimes" des exclusions de cette politique. Les différents plans nationaux (Premier Plan Triennal, Premier et Deuxième Plan Quadriennaux) ainsi que le Programme spécial de la wilaya de Saïda qui couvraient une grande partie de la steppe, n'ont pas pu sortir la région de son retard par rapport à d'autres régions du pays. La répartition géographique des investissements n'était pas optimale, puisque le nord de la wilaya de Saïda s'est accaparé la majorité des projets industriels. Les quelques investissements que la steppe a pu récupérer dans le domaine de l'habitat et l'éducation essentiellement, ont été absorbés par les villes importantes de la région au détriment des zones rurales. Après 1985, la steppe occidentale a été bouleversée sur le plan administratif, avec en premier lieu, la création de deux wilayas et en second lieu, le doublement du nombre de ses communes. Cette action de maîtrise effectuée par l'Etat, a eu un rôle appréciable dans l'assistance et l'encadrement de la population pastorale en matière de services, administratifs et éducatifs essentiellement. Après cette date, l'école est devenue accessible pour la majorité des enfants de la steppe, notamment pour les enfants nomades. Ce début d'équipement de la steppe occidentale n'a pourtant pas été suivi par un développement économique, les quelques implantations industrielles reçues étant trop ponctuelles et concernant quelques villes de la steppe. En fait, dans la steppe, la majorité des programmes sectoriels, ont surtout concerné l'espace physique (boisement le long des routes, boisement autour des agglomérations, lutte contre la désertification...). 
Malgré des efforts considérables dans le domaine des services sociaux et de la scolarisation essentiellement, et dans celui du désenclavement par la création de nouveaux axes routiers, tout reste à faire semble-t-il dans la steppe. Le domaine majeur est le secteur primaire où la politique de mise en valeur agricole tend à prendre de l'ampleur au détriment d'une activité pastorale en difficulté et où le processus de désertification de la steppe occidentale est en train de prendre une dimension écologique inquiétante capable de compromettre toute tentative de développement de cet espace... (Hadeid, 2006)

\section{Glossaire et sigles :}

Achaba : transhumance entre le piémont saharien et les hautes terres.

Chott: auréole de végétation halophile entourant une dépression fermée; par extension, lac salé.

Daïra: Circonscription administrative (correspond à l'arrondissement).

Erg: massif dunaire.

Gourbi : Habitation rudimentaire faite de branchages et de terre sèche

Ksourienne : Ksar (pl. ksour): village traditionnel, se situant au niveau de l'Atlas Saharien.

Wilaya : circonscription administrative (correspondant au département).

BAE : Branche d'activité économique.

BTP : Bâtiment et travaux publics.

CEM : Collège d'enseignement moyen.

ONS : Office national des statistiques.

PNDA : Plan national du développement de l'agriculture.

RGPH : Recensement général de la population et de l'habitat.

SONIC : Société nationale des industries de cellulose.

PSW : Programme spécial de wilaya.

\section{BIBLIOGRAPHIE}

Bounouara A., 1981. Suburbanisation d'une agglomération rurale dans la daïra de Saïda: le cas de Rebahia. Mémoire de D.E.S, Géographie, Université d'Oran, 76 pages.

Couderc R., 1979. Géographie et développement: Les Hautes Steppes occidentales, Thèse d'État, Montpellier III, 655 pages.

Hadeid M., 1996. Croissance et développement de petites villes et leur rôle dans l'organisation de l'espace de la steppe occidentale (Algérie occidentale). Magister, Géographie, Université d'Oran, 209 pages.

Hadeid M., 2002. Difficultés d'intégration d'une région à l'espace national, le cas des Hautes Plaines occidentales (L'Ouest algérien), Bulletin des Sciences Géographiques, n 10, INCT, Alger, pp. 07-15. 
Hadeid M. 2006. Les mutations spatiales et sociales d'un espace à caractère steppique, le cas des Hautes Plaines occidentales (Algérie), Thèse de Doctorat d'Etat en Géographie, Thèse soutenue dans le cadre de la co-tutelle entre l'Université d'Oran (Algérie) et l'Université de Franche-Comté (France), 506 pages.

Hadeid M. 2008. Approche anthropique du phénomène de désertification dans un espace steppique : le cas des hautes plaines occidentales algériennes, Vertigo - la revue électronique en sciences de l'environnement, Volume 8 Numéro 1 | avril 2008, [En ligne], mis en ligne le 07 novembre 2008. URL : http://vertigo.revues.org/index5368.html

\section{NOTES}

1. R.G.P.H. de 1966, caractéristiques socio-économiques, Série: A, Volume: VI, S.E.P., Alger, 1971.

\section{RÉSUMÉS}

L'Algérie a connu des mutations importantes grâce au programme de développement entrepris par l'Etat et ce, dès l'indépendance. Ce projet avait un double objectif : hisser le pays à un certain degré de développement économique et social et améliorer les conditions de vie des Algériens. Cependant, cette politique a beaucoup évolué au cours de l'histoire. En effet, l'intervention de l'Etat à travers sa politique d'industrialisation et de découpages administratifs n'a pas pu faire disparaître les disparités régionales héritées de la période coloniale. Les Hautes Plaines occidentales demeurent toujours en marge du développement. La politique d'aménagement du territoire du pays n'a fait qu'accentuer les déséquilibres déclenchés durant la période coloniale et ce, malgré les efforts consentis en matière d'équipement et de désenclavement. Le problème de dégradation de la steppe et la crise de l'activité pastorale en témoignent.

Algeria has experienced important changes thanks to the development programme undertaken by the state from the independence. This project had dual purpose: Raising the country to economic and social level and improving living conditions of Algerian. However, this policy has undergone some slight changes throughout time as well as in level space. In fact, the interference of the state through industrialization policy and administrative apportionments were unable to bring to end regional disparities inherited from colonial period. Western high plains, for example, are still not benefiting from development. Regional and country planning policy has accentuated the imbalances which begun during colonial period and yet, the approved efforts as far as equipment and Opening up. The crisis presented by the degradation of the steppes and other pastoral activities is certainly the best evidence. 


\section{INDEX}

Mots-clés : développement, industrialisation, découpages administratifs, disparités régionales, aménagement du territoire, désenclavement, dégradation, activité pastorale

Keywords : development, industrialization, administrative apportionments, regional disparities, regional and country planning, opening up, degradation, pastoral activity

\section{AUTEUR}

\section{MOHAMED HADEID}

Mohamed Hadeid est Maître de Conférences au département de géographie et de l'aménagement du territoire (Université d'Oran, Algérie) et membre du laboratoire EGEAT, Chercheur-associé au CRASC, Algérie. Membre de l'Observatoire sur les Hautes Plaines (ROSELT). hadidom@yahoo.fr 